\title{
Verunsicherte Mitte, gespaltene Gesellschaft?
}

\begin{abstract}
Die gegenwärtige Diskussion um die Zukunft der Einkommensmitte muss erweitert werden um eine Diskussion über die Ursachen und Folgen der sich ausbreitenden Ungleichheit und Unsicherheit. Die entscheidende Frage ist die nach den zukünftigen Absicherungen der Lebensperspektiven aller Mitglieder der Gesellschaft, ohne die Demokratie nicht möglich ist. Wie die lohn- und gehaltsabhängige Mittelklasse auf die eigenen Unsicherheiten und Verunsicherungen reagiert, wird eine wichtige Rolle dabei spielen, ob sie für eine Politik der Reduzierung sozialer Ungleichheit gewonnen werden kann. ${ }^{1}$
\end{abstract}

\section{Die Legitimität der sozialen Ungleichheitsverhältnisse steht infrage}

$\mathrm{Ob}$ und wieweit ein Gemeinwesen als demokratisch bezeichnet werden kann, lässt sich nicht allein an seiner politischen Verfassung, der Balance wechselseitiger Kontrollen durch Institutionen und den rechtlich zugestandenen Möglichkeiten seiner Bürgerinnen und Bürger ermessen, Einfluss auf die Gesetzgebung auszuüben. Wesentlich hinzukommen muss ein sozialmaterieller „Unterbau“, der die Menschen in die Lage versetzt, bereits im Alltag, in und außerhalb ihrer Arbeit sowie in ihrer gesamten Lebensführung mit guten Gründen (und nicht nur aus Zwängen heraus) Entscheidungen zu treffen und ihr Leben für sich und mit anderen zu gestalten (Sen 2002, S. 29ff.). Wem diese Erfahrung und die dazu notwendigen materiellen Sicherheiten verweigert werden, dem fehlen auch die Mittel, sich als Bürger zu verstehen und zu behaupten. Was soll jemand von „der Politik" erwarten, der oder die sich bereits im alltäglichen Leben als ohnmächtig erfährt? Fragen von sozial-materieller Absicherung und Unsicherheit werden somit zu Kernfragen der Demokratie. Sie betreffen zugleich die Legitimität sozialer Ungleichheit, also den Tatbestand, dass es in der Gesellschaft ungleiche Zugangsmöglichkeiten zu Ressourcen gibt, die Sicherheiten gewährleisten.

Was bedeutet es dann für eine Gesellschaft, die sich der politischen Verfassung nach als Demokratie begreift, wenn die Mehrheit ihrer Bevölkerung den Ungleichheitsverhältnissen, in denen sie lebt, und somit dem sozial-materiellen Unterbau zunehmend die Legitimität abspricht? Genau das scheint in der Bundesrepublik Deutschland mittlerweile der Fall zu sein. In einer im letzten Herbst veröffentlichten Umfrage von Wissenschaftlern der HumboldtUniversität bewerteten über $80 \%$ der Befragten die Einkommens- und Vermögensverteilung in der Bundesrepublik Deutschland als ungerecht. Nur noch Wenige noch nicht einmal ein Drittel - sahen eine Chancengleichheit in der Bildung, in den Möglichkeiten für sozialen Aufstieg und für Selbstverwirklichung gegeben. Dass Leistungsgerechtigkeit verwirklicht sei, meinte gerade noch die Hälfte der Befragten, ein Fünftel weniger als noch Anfang der 1990er Jahre (Bigalke 2007). Einer von der Bertelsmannstiftung im Dezember letzten Jahres publizierten Umfrage zufolge gaben nur $15 \%$ der Befragten an, der Aufschwung komme tatsächlich bei ihnen an, und nur noch $5 \%$ hielten die Bundesrepublik für das entwickelte Industrieland, das ihren Vorstellungen von sozialer Gerechtigkeit am nächsten kommt. $57 \%$ sahen hingegen die skandinavischen Länder als Vorbild an (Spiegel online 2007).

Diese Befunde drücken mehr als eine momentane Stimmungslage aus. Bereits die im Datenreport des Statistischen Bundesamts in den letzten Jahren wiedergegebenen Ergebnisse zu Gerechtigkeitsfragen wiesen in die gleiche Richtung: Weniger als $40 \%$ der Westdeutschen und kaum $20 \%$ der Ostdeutschen hielten im Jahr 2004 die sozialen Unterschiede für gerecht, die Mehrheit (eine knappe im Westen, eine deutlichere im Osten des Landes) ging davon aus, dass die Ungleichheit weiter reduzierbar sei (Statistisches Bundesamt 2006, S. 626).

Begleitet wird die vorherrschende Verurteilung der Ungleichheitsverhältnisse von einer sich ausbreitenden Verunsicherung. Sie ist am stärksten ausgeprägt in der Arbeiterschaft, reicht mittlerweile aber auch in Abstufungen weit in die mittleren
Sozial- und Einkommenslagen hinein. Verunsicherung entsteht insbesondere mit Blick auf das Einkommen, den Arbeitsmarkt, die Alterssicherung, die Zukunftsperspektiven der Kinder (Böhnke 2006, S. 126; Vogel 2006). In einer Studie der Friedrich-Ebert-Stiftung aus dem Jahr 2006 gaben 63 \% der Befragten an, ihnen machten die gesellschaftlichen Veränderungen Angst, annähernd die Hälfte empfand das eigene Leben als ständigen Kampf, $59 \%$ berichteten, sie müssten sich derzeit finanziell einschränken und $49 \%$ befürchteten, ihren Lebensstandard nicht halten zu können (Friedrich-Ebert-Stiftung 2006). Es passt in das Gesamtbild, wenn annähernd zeitgleich eine Untersuchung des DGB erhebliche Unzufriedenheiten mit den Arbeitsverhältnissen zutage fördert. Demnach beurteilt ein Drittel aller Beschäftigten die eigenen Arbeitsbedingungen als schlecht, nur $12 \%$ sind mit ihrer Arbeit zufrieden (Kazim 2007).

Nimmt man diese Befunde ernst und betrachtet sie in ihrem Zusammenhang, dann verweisen sie auf der subjektiven Ebene der Wahrnehmung von Gesellschaft und der eigenen Stellung in ihr auf eine Erosion der sozial-materiellen Grundlagen von Demokratie. Diese Wahrnehmung ist selbst ein gesellschaftlicher Tatbestand und als

1 Für wichtige Anregungen und Hinweise danke ich
Ulf Kadritzke.

Martin Kronauer, Dr. phil., habil., ist Professor für Gesellschaftswissenschaft an der Fachhochschule für Wirtschaft Berlin. Arbeitsschwerpunkte: Strukturwandel und Wohlfahrtsstaaten im internationalen Vergleich, Prekarität und Exklusion, Armut. e-mail: kronauer@fhw-berlin.de 
solcher bedeutsam und wirkmächtig. Dass sich die Verunsicherungen und Ungerechtigkeitserfahrungen wieder rasch verflüchtigen dürften, etwa im Zuge eines anhaltenden Wirtschaftswachstums, erscheint unrealistisch. Dazu liegen die Gründe zu tief und weisen die Ungerechtigkeitserfahrungen bereits eine zu hohe Stabilität auf.

\section{2 \\ Vermeidung und Engführung der politischen Auseinandersetzung mit Ungleichheit}

Eine ernsthafte Auseinandersetzung mit der zunehmenden sozialen Ungleichheit, ihren subjektiven Verarbeitungen und gesellschaftspolitischen Implikationen findet gleichwohl auf der politischen Ebene nicht statt. Ungleichheitsthemen tauchen zwar in den Medien und der politischen Debatte regelmäßig auf, wenn wieder einmal eine Studie einschlägige Zahlen vorlegt. Sie erregen für kurze Zeit die Gemüter, um dann bald darauf von der Bildfläche der auf Kaufkraft und Wählerstimmen hin organisierten "Öffentlichkeit“ zu verschwinden. So war es mit der „Unterschichtdebatte“ im Herbst 2006, so ist es bei jeder Veröffentlichung eines neuen Armuts- und Reichtumsberichts der Bundesregierung, und so dürfte es auch um die gerade stattfindende Diskussion um die Zukunft der „Mitte“ bestellt sein.

Angestoßen wurde diese Debatte durch Untersuchungen des Deutschen Instituts für Wirtschaftsforschung (DIW) Berlin zur Einkommensverteilung. Sie wiesen nach, dass seit dem Ende der 1990er Jahre in Deutschland die Bevölkerung mit mittleren Einkommen abnimmt, dass dabei die Einkommensaufstiege deutlich schwächer ausfallen als die Abstiege, womit eine Polarisierung der Einkommen zulasten der gesellschaftlichen „Mitte“ stattfindet (Grabke/Frick 2008). Auffallend ist der politische Reflex der Parteien, das Problem sogleich wieder auf eine Diskussion über eine Steuerentlastung für mittlere Einkommen engzuführen. Wenn die eingangs aufgeführten Anzeichen nicht trügen, greift eine solche Debatte nicht nur zu kurz, sie entzieht geradezu die wichtigen Fragen dem Blick.

$\mathrm{Zu}$ den entscheidenden Fragen gehört die nach den zukünftigen Absicherungen der Lebensperspektiven und damit der gesellschaftlichen Teilhabemöglichkeiten, von denen eine Demokratie lebt. Das ist keineswegs eine spezielle Mittelschichtsoder gar Mittelstandsfrage. Sie betrifft vielmehr die Ungleichheitsverhältnisse in der ganzen Gesellschaft, damit vor allem und am dringlichsten diejenigen Menschen, die am härtesten von sozialer Ungleichheit betroffen sind, weil sie kaum oder gar nicht über Ressourcen der Mittelschicht (Bildungszertifikate und Qualifikationen, tragfähige soziale Netze, häufig auch kleinere oder größere Vermögen) verfügen.

Allerdings kommt der gesellschaftlichen „Mitte“ insofern eine politisch besondere Bedeutung zu, als sie die Mehrheit der Bevölkerung umfasst und bislang von den sozialstaatlichen Absicherungen besonders profitierte. Wird diese - in sich sozial sehr heterogene - Mitte bereit sein, nach neuen, solidarischen und somit über die „Mitte“ hinausreichenden Antworten auf die sich ausbreitenden sozialen Unsicherheiten und Ungleichheiten im Rahmen sozialstaatlicher Leitbilder zu suchen? Oder wird sie sich weiter in unterschiedliche Interessenlagen aufspalten, um jeweils, ,auf eigene Faust" (zum Beispiel mit den Mitteln der Steuerpolitik) Antworten auf ihre je besonderen Verunsicherungen und Ungerechtigkeitserfahrungen $\mathrm{zu}$ finden und durchzukämpfen, im Zweifelsfall auch gegen die bereits sozial Absteigenden und Deklassierten? In diesem Sinn hängt es also in der Tat erheblich von Reaktionen in der gesellschaftlichen Mitte ab, ob die gesellschaftlichen Ungleichheiten weiter vertieft oder eher zurückgenommen werden, ob der sozial-materielle Unterbau von Demokratie weiter geschwächt oder eher gestärkt wird.

\section{Die lohn- und gehalts- abhängige "Mitte" der Sozialstaat und die Demokratie}

Wer zur gesellschaftlichen „Mitte“ gehört und was diese kennzeichnet, stellt sich je nach dem gewählten analytischen Zugang anders dar. Die „Mittelschicht“ kann als Einkommensklasse, als sozialstrukturelle Kategorie, aber auch als sozial zugeschriebene und gefühlte Realität beschrieben und verstanden werden. Alle drei Gesichtspunkte tragen zur Klärung der Frage nach den Verunsicherungen der gesellschaftli- chen Mitte und ihren gesellschaftspolitischen Implikationen bei, unter allen drei soll die Mitte deshalb zunächst kurz charakterisiert werden.

\subsection{DIE EINKOMMENSMITTE}

Die Mitte, von der gegenwärtig vor allem die Rede ist, wird allein durch das Einkommen definiert. Das DIW ordnet der Einkommensmittelschicht alle Personen in Privathaushalten mit einem bedarfsgewichteten Nettoeinkommen zwischen $70 \%$ und $150 \%$ des mittleren Einkommens (Median) zu. Deren Anteil nahm von $62 \%$ im Jahr 1999 auf 54 \% im Jahr 2006 ab (Grabke/ Frick 2008, S. 102f.). Mit diesem Befund einer vor allem durch soziale Abstiegstendenzen „schrumpfenden Mittelschicht“ wurde deutlich, dass die wachsende Einkommensungleichheit im Zentrum der Gesellschaft angekommen ist.

\subsection{DIE SOZIALSTRUKTURELLE MITTE}

Soziologen haben sich mit der Bestimmung der Mittelpositionen in der Sozialstruktur von Industriegesellschaften immer recht schwer getan, seitdem diese Position nicht mehr vor allem vom „Mittelstand" der selbstständigen Handwerker, Landwirte, kleinen Gewerbetreibenden und freien Berufe sowie den in verschiedener Hinsicht privilegierten Beamten besetzt wurde. Mit der Entwicklung der Großindustrie und der Ausdifferenzierung von Unternehmerfunktionen in eigenständigen betrieblichen Abteilungen, dem Ausbau der öffentlichen Verwaltungen und Dienstleistungen in der zweiten Hälfte des 19. Jahrhunderts bildete sich die „mittlere Lage“ der „Lohn- und Gehaltsbezieher höherer Qualifikation“ (Geiger 1932/1967, S. 24) heraus, bisweilen auch als die „neue Mittelklasse" bezeichnet.

Von den Lagemerkmalen her nahm und nimmt die sozialstrukturelle Mitte eine Zwitterstellung ein, die in Deutschland auch rechtlich, etwa in der Statusunterscheidung von Arbeitern, Angestellten und Beamten, festgeschrieben wurde. Mit den Arbeitern teilen die Angehörigen der neuen Mittelklasse die Eigentumslosigkeit von Kapital (zumindest können sie von den Kapitalanteilen, sollten sie solche besitzen, nicht leben). Von den Arbeitern unterscheidet sie jedoch die weitgehende Befreiung von manueller Arbeit, der Umfang und das Profil der Qualifikationen (des 
verinnerlichten „kulturellen Kapitals“, um einen Begriff Bourdieus aufzunehmen, das sie vor allem von den un- und angelernten Arbeitern absetzt), Sonderregelungen der Arbeitsverhältnisse, Sozialversicherungen und Bezahlung, oft auch eine gehobene Stellung in der Betriebs- oder Verwaltungshierarchie, verbunden in bestimmten Positionen mit Anweisungs- und Kontrollfunktionen gegenüber Untergebenen (Wright 1997, S. 19ff.). In sich ist die neue Mittelklasse äußerst uneinheitlich. Die Übergänge zwischen Facharbeitern und einfachen Angestellten waren immer schon fließend, viele (gerade rechtliche) Grenzziehungen waren willkürlich, sollten die Lohnabhängigen spalten und sind heute obsolet. Am anderen Pol, bei den leitenden Angestellten im oberen Management, sind die Übergänge zu den Kapitaleignern ebenfalls unscharf. Besonders in der „Mitte der neuen Mittelklasse" aber bleibt die Zwitterstellung deutlich erkennbar. Bourdieu und andere haben hierfür den Begriff der „Gehaltsempfänger-Bourgeoisie“ "geprägt, zu der sie unter anderem Ingenieure, Wissenschaftler und Studienräte zählen (Bourdieu et al. 1981, S. 50).

Gerade aus dieser Zwitterstellung resultiert eine Uneindeutigkeit der Interessenlagen, gesellschaftlichen Selbstverortungen und politischen Orientierungen. Soziologinnen und Soziologen hat diese Uneindeutigkeit immer schon fasziniert (nicht zuletzt deshalb, weil sie selbst dieser Mittelklasse angehören) und sie versuchen, ihr mit Untersuchungen zu „Mentalitäten“ (Geiger) oder „Habitus“ (Bourdieu) auf die Spur zu kommen. Verfehlt wäre es, aus der „Mittellage“ auf eine besondere Affinität zur Demokratie zu schließen. Im historischen Rückblick betrachtet, waren die Mittelklassen der Weimarer Republik - die "alte“ wie die „neue“ - mehrheitlich ebensowenig wie das Großbürgertum ein Rückhalt der Demokratie (vgl. hierzu auch Demirović in diesem Heft). Insofern hat die politische und kulturelle Demokratisierung der „Mitte“ in Deutschland in einer widersprüchlichen und konfliktreichen Geschichte vor allem in der Nachkriegszeit stattgefunden, wobei nicht zuletzt dem Sozialstaat eine bedeutende Rolle zukam.

\subsection{DIE GEFÜHLTE MITTE}

Die deutliche Mehrheit der westdeutschen Bevölkerung, zwischen $55 \%$ und knapp über $60 \%$, ordnet sich selbst regelmäßig der Mittelschicht zu (Statistisches Bundesamt 2006). In Ostdeutschland dominierte unmittelbar nach der „Wende“ in gleichem Umfang die Einstufung in die Arbeiterschicht, danach verschob sich auch dort die gefühlte Zugehörigkeit mehrheitlich zur Mitte, wenngleich diese Zuordnung noch nicht so gefestigt ist wie in den alten Bundesländern.

Wie die ursprüngliche Ost-West-Differenz zeigt, sind soziale Selbsteinstufungen stark von dem Gesellschaftsbild geprägt, das in einer Gesellschaft propagiert und institutionell gestützt wird. Das in Westdeutschland lange Zeit vorherrschende Bild, in einer um eine Mitte zentrierten, somit nicht polarisierten Gesellschaft zu leben, wäre ohne das Wirtschafts- und Beschäftigungswachstum der Jahrzehnte nach dem Zweiten Weltkrieg und ohne den damit einhergehenden Ausbau sozialstaatlicher Sicherungssysteme und Dienstleistungen nicht denkbar. ${ }^{2}$ Die gefühlte „Mitte" ist somit in hohem Maße ein Produkt des Sozialstaats.

Dies gilt nicht nur mit Blick auf die im öffentlichen Dienst geschaffenen Arbeitsplätze und die Ausweitung seiner Dienstleistungen, wie etwa des Bildungswesens. Es gilt vor allem auch wegen der Sicherheiten, die sozialstaatliche Regelungen für die Lebensplanung schufen. War eine relative Sicherheit der Lebensumstände zuvor ein Privileg der Besitzer von Vermögen und Kapital, so organisierte der Sozialstaat nun (nach einer langen Geschichte sozialer Kämpfe) ein „Sozialeigentum“ (Castel 2000, S. 264), das die eigentumslose, lohnabhängige Mehrheit mit Solidarleistungen gegen die am meisten verbreiteten Risiken der Einkommenslosigkeit absichern sollte. Finanziert wird es durch die eigene Erwerbsarbeit, somit gewissermaßen aus eigener Tasche. Das grundlegende Machtgefälle zwischen Kapitaleignern und Lohnabhängigen wurde damit zwar nicht außer Kraft gesetzt, aber durch die Einbindung der Interessenvertretungen der Lohnabhängigen in die intermediären Institutionen des Sozialstaats abgeschwächt. Ebensowenig wurden die grundlegenden Unsicherheiten beseitigt, die von Marktabhängigkeit und Kapitaldominanz ausgehen. Aber sie blieben in der Phase relativer Vollbeschäftigung latent, und vor allem das Gespenst der Arbeitslosigkeit verlor für eine Weile seinen Schrecken.

Die Absicherung der Lebensverläufe so weit wie immer möglich - gegen Un- wägbarkeiten, die von Marktabhängigkeiten ausgehen, ist nicht in erster Linie eine versicherungstechnische Angelegenheit, wie es derzeit meist diskutiert wird. Sie gehört vielmehr, wie Kaufmann unterstreicht, zur „Gewährleistung sozialer Grundrechte" (Kaufmann 2003, S. 100). Damit zählt sie zu den sozial-materiellen Voraussetzungen von Demokratie in einem doppelten Sinn: Die Institutionalisierung von „sozialer Sicherheit" schafft ein gewisses Gegengewicht gegen die Übermacht der Kapitalseite. Und erst „die Gewährleistung von Lebensperspektiven für die einer politisch verfassten Gesellschaft Zugehörigen “ (Kaufmann 2003, S. 101) eröffnet Letzteren die Möglichkeit der Teilhabe in den verschiedenen Sphären des gesellschaftlichen Lebens, oder kurz gesagt: gesellschaftliche „Inklusion“ (Kaufmann 2003, S. 100).

Lebensperspektiven wurden im westdeutschen Sozialstaat lange Zeit durch eine „Standardisierung von Lebensverläufen “ (Kaufmann 2003, S. 101) sichergestellt, mit dem im Nachhinein so bezeichneten Normalarbeitsverhältnis (unbefristete Vollzeitbeschäftigung mit Tariflöhnen, die den Unterhalt einer Familie gewährleisten sollten) im Zentrum. Nicht auf die Beseitigung sozialer Ungleichheit waren und sind die beitragsfinanzierten Sicherungssysteme angelegt, sondern auf Sicherheiten auf der Basis von Unterschieden der Erwerbsposition und des sozialen Status. Das machte den deutschen Sozialstaat auch für statusbewußte Mitglieder der „neuen Mittelklasse“ attraktiv. Gerade in dieser engen Verbindung von sozialen Rechten und Erwerbspositionen liegt aber auch die Achillesferse des korporatistischen Sozialstaats deutscher Prägung. Denn mit hoher Arbeitslosigkeit und zunehmender Entstandardisierung der Erwerbsarbeit gerät dann auch dieses Gefüge sozialstaatlicher Gewährleistungen von Lebensperspektiven ins Rutschen, wie wir es heute beobachten (vgl. auch Schmid und Trampusch in diesem Heft).

\footnotetext{
2 Der Befund aus der Befragung im Auftrag der Friedrich-Ebert-Stiftung aus dem Jahr 2006, demzufolge 69 \% der Bevölkerung der Aussage zustimmten, es gebe keine gesellschaftliche Mitte mehr, nur noch ein "Oben" und ein "Unten" (Friedrich-Ebert-Stiftung 2006), deutet darauf hin, dass sich dieses Gesellschaftsbild ändern könnte. Siehe hierzu Neckel 2008.
} 
In der wachsenden Einkommensungleichheit zeigt sich eine massive Verschiebung in den Kräfteverhältnissen zwischen Kapital und Arbeit, die sich inzwischen auch deutlich auf die lohn- und gehaltsabhängige Mitte auswirkt. Als „Exportweltmeister" hat sich Deutschland nur auf der Grundlage lange Zeit stagnierender Reallöhne etablieren können. Produktivitätsgewinne wurden zunehmend zugunsten der Gewinneinkommen verteilt, dagegen sank der Anteil stetig, den die abhängig Erwerbstätigen am Volkseinkommen für sich verbuchen konnten. Dazu hat die Steuerpolitik wesentlich beigetragen (Andreß/ Kronauer 2006, S. 38f.). Die den öffentlichen Haushalten auferlegte Sparpolitik tat ein Übriges, die Löhne und Gehälter zu deckeln. Dass ein solches Verteilungsmuster möglich wurde, ist nicht zuletzt auf die Schwächung der Verhandlungsmacht der Gewerkschaften durch die anhaltend hohe Arbeitslosigkeit und die Drohgebärden von Unternehmen, Standorte zu verlagern, zurückzuführen. Im wachsenden Dienstleistungsbereich ist der Organisationsgrad ohnehin niedriger und auch deshalb die Einkommenspreizung größer. Vor allem dort hat sich mittlerweile - forciert durch die Gesetzgebungen der Arbeitsmarktreform - ein Niedriglohnbereich festgesetzt, der die Einkommenskluft weiter vertieft.

Stagnierende oder sinkende Realeinkommen in der Einkommensmitte etablierten sich zeitgleich mit exorbitanten $\mathrm{Zu}$ wächsen der Einkommen im Topmanagement seit Mitte der 1990er Jahre. Die Demonstration, dass es im vom Finanzmarkt dominierten Kapitalismus der Gegenwart weniger denn je einen gemeinsamen Maßstab gibt, an dem sich sowohl die Manager und deren Gehälter als auch die abhängig Beschäftigten und deren Einkommen messen lassen müssen, hätte kaum deutlicher ausfallen können. „Leistung“, ein ohnehin problematischer Vergleichsmaßstab, wird vollends illusorisch, wenn die einen auf einen Schlag das Vielfache, die anderen aber noch immer bestenfalls das Gleiche verdienen. Damit gerät aber auch eine gerade im Selbstverständnis von Angehörigen der neuen Mittelklasse zentrale Säule der Legitimation sozialer Ungleichheit ins Wanken, die Leistungsgerechtigkeit (Kronauer 2007).

\subsection{FLEXIBILISIERUNGSZWÄNGE IN DER ERWERBSARBEIT}

Auf den ersten Blick machen die Veränderungen in den Arbeits- und Beschäftigungsverhältnissen in Deutschland den Eindruck eines eher gemäßigten Wandels. Denn auch heute noch arbeitet über die Hälfte der Lohn- und Gehaltsabhängigen in unbefristeter Vollzeitbeschäftigung (Alda 2005, S. 249). Dort, wo es offensichtlich problematisch wird, bei den befristeten Beschäftigungsverhältnissen, der Zeitarbeit und in vielen Fällen auch bei den „Alleinselbstständigen“, steigen die Zuwächse zwar zum Teil dramatisch an, die absoluten Zahlen und relativen Anteile an der Gesamtbeschäftigung sind bis jetzt aber noch immer recht bescheiden. Letztere bewegen sich jeweils in einem Bereich von mehr oder weniger deutlich unter $10 \%$ (Keller/ Seifert 2007; Schmid in diesem Heft).

Der erste Eindruck ist nicht falsch, aber unvollständig - deshalb doch trügerisch. Das zeigt sich zum einen im Zeitverlauf, zum anderen beim Blick in die Arbeitssituationen hinein.

\section{ERWERBSBIOGRAFIEN WERDEN BRÜCHIGER}

Im Zeitverlauf wird sichtbar, dass die Erwerbsbiografien rasch und in erheblichem Umfang brüchiger werden. Je jünger der Jahrgang, desto häufiger die Erfahrung mit Arbeitslosigkeit vor dem 30. Lebensjahr. Bereits über die Hälfte der zwischen 1961 und 1965 Geborenen machte diese Erfahrung, in den Jahrgangsgruppen zehn Jahre zuvor waren es erst $30 \%$, noch einmal zehn Jahrgänge zurück weniger als $10 \%$. Gleichzeitig nahm der zeitliche Umfang der Arbeitslosigkeit in den Erwerbsbiografien zu (Klammer 2007, S. 255f.). Vor allem der Einstieg ins Berufsleben ist damit schwieriger geworden, was wiederum auf das spätere Einkommen und die sozialversicherungsrechtlichen Ansprüche ausstrahlt. Dies betrifft auch die neue Mittelklasse und deren Kinder. Befristete Arbeitsverhältnisse, die für die Einstiegsphase immer wichtiger werden, sind keineswegs auf gering qualifizierte Tätigkeiten beschränkt, sondern finden sich auch bei Beschäftigten mit akademischem Abschluss.

Bemerkenswert ist dabei vor allem der Langzeiteffekt von Befristungen. Die Einkommensnachteile, die aus befristeter Beschäftigung resultieren, werden selbst beim 
späteren Übergang in unbefristete Arbeitsverhältnisse nicht mehr vollständig ausgeglichen (Gross 2007, S. 164). Auch hier sind mit dem biografischen Unsicherheitsmoment zugleich Gerechtigkeitsprinzipien betroffen. „Strukturelle Privilegierung und differenzierte Mobilitätschancen" aufgrund von Unterschieden in der rechtlichen Absicherung von Arbeitsverhältnissen „widersprechen dem ,Equity Prinzip“ und dem Prinzip der Chancengleichheit" (Groß 2007, S. 164).

\section{RISIKEN INTERNER FLEXIBILISIERUNG}

Trügerisch ist aber auch die Vorstellung von Stabilität, die sich daran festmacht, dass unbefristete Vollzeitarbeit noch so weit verbreitet ist. Denn in der Hülle des sogenannten Normalarbeitsverhältnisses haben drastische Veränderungen in den Arbeitsbedingungen stattgefunden, die erhebliche Risiken auch für die qualifizierten und hochqualifizierten Lohn- und Gehaltsabhängigen mit sich bringen. Das einschlägige Stichwort hierzu ist ,interne Flexibilisierung".

Im Unterschied zu Strategien der „externen Flexibilisierung", bei denen Unternehmen und andere Organisationen je nach Bedarf Arbeitskräfte entlassen oder einstellen und dabei die Mittel der Befristung und Zeitarbeit nutzen, beruhen Stragien der „,internen Flexibilisierung “ darauf, die Arbeitszeiten und Arbeitszuordnungen einer gegebenen Belegschaft flexibel zu gestalten und unter Umständen auch die Entlohnung stärker zu variieren. Auf diese Weise sollen Marktschwankungen aufgefangen, aber auch wechselnde Vorgaben der Unternehmensspitze umgesetzt werden (Keller/Seifert 2007).

Strategien der internen Flexibilisierung werden häufig mit Formen der indirekten Unternehmenssteuerung verbunden, also der Steuerung mit Hilfe von Kennziffern, die aus dem Vergleich mit externen oder intern eigens geschaffenen Konkurrenten gewonnen werden. Damit verwischen die Grenzen zwischen Hierarchie und Markt. Innerhalb der Hierarchie selbst sind nun die Beschäftigten sehr viel unmittelbarer mit externen oder künstlich inszenierten internen Marktrisiken konfrontiert. Hierarchisch ausgeübte Herrschaft verbirgt sich zunehmend hinter der scheinbaren Objektivität von neutralen Marktzwängen. Flachere Hierachien versprechen zwar einen Bürokratieabbau, reduzieren aber zugleich
Aufstiegsmöglichkeiten und geraten damit in Konflikt mit den Karriereorientierungen innerhalb der abhängigen „Mitte“.

Die soziologische Arbeitsforschung weist vor allem auf vier Arten von Risiken und damit Quellen der Verunsicherung hin, die mit Strategien der internen Flexibilisierung und der Einführung marktförmiger Steuerungselemente verbunden sind: (1) Arbeitszeitrisiken, (2) Gesundheitsrisiken, (3) Einkommensrisiken, (4) Beschäftigungsrisiken (Flecker 2007, S. 86).

(1) Arbeitszeitrisiken: Sie erwachsen daraus, dass Arbeitszeitregelungen immer mehr entstandardisiert, von der tariflichen auf die betriebliche Ebene verschoben und im Extremfall individuell ausgehandelt werden. Die problematische Kehrseite ist, dass der Schutz einforderbarer Zeitgrenzen zunehmend aufgeweicht wird oder gar entfällt. Zielvereinbarungen räumen den Beschäftigten auf der mittleren und höheren Hierarchiebene größere Eigenverantwortlichkeiten für die Durchführung ihrer Arbeitsaufgaben ein. Aber die dabei immer wieder höher gelegte Meßlatte der Gewinnbzw. Einsparungsvorgaben erhöht zugleich den Leistungsdruck, der zu selten organisatorisch aufgefangen und stattdessen direkt an die Beschäftigten weitergegeben wird. Flexible Arbeitszeiten, Leistungsvereinbarungen und Vertrauensarbeitszeitregelungen erhöhen vor allem dann die Gefahr der Arbeitsüberlastung, wenn die Unternehmen, wie es vielfach der Fall ist, eine „Personalpolitik der unteren Linie“ fahren, sich also bei der Personalausstattung an einer schwachen Auslastung orientieren und damit die Personalressource verknappen (Lehndorff 2007, S. 208). Zudem treibt die permanente Aufforderung, sich auch innerhalb des Unternehmens als Marktsubjekt zu verstehen, das den Risiken der Konkurrenz ausgesetzt ist, dazu, die noch geltenden Regeln der Arbeitszeitbegrenzung selbst zu überschreiten, auch auf Kosten der eigenen Lebenszeit und Lebensqualität jenseits der Erwerbsarbeit (Kadritzke 2004; Schumann in diesem Heft).

(2) Gesundheitsrisiken: Mit der Intensivierung der Arbeit, verstärktem Zeit- und Leistungsdruck nehmen die psychosomatischen Gesundheitsrisiken zu. Psychosomatische Störungen gehören mittlerweile zu den häufigsten Ursachen bei Krankmeldungen. Inbesondere in den Gesundheitsberufen und der öffentlichen Verwaltung breiten sie sich in Deutschland aus. Vom "erschöpften Selbst" spricht der französische Soziologe Ehrenberg, um die neue Qualität des psychischen Leidens in der Gegenwartsgesellschaft zu kennzeichnen. „Das ideale Individuum wird nicht mehr an seiner Gefügigkeit gemessen, sondern an seiner Initiative" (Ehrenberg 2004, S. 9). Die Erschöpfung ist die Folge einer permanenten Überforderung, nämlich der „Aufgabe, alles zu wählen und alles zu entscheiden" (Ehrenberg 2004, S. 222) - ohne allerdings über die notwendigen Entscheidungsvoraussetzungen zu verfügen. Dies gilt heutzutage auch und gerade für Arbeitssituationen: „Das Unternehmen ist das Vorzimmer der nervösen Depression geworden“" (Ehrenberg 2004, S. 221).

(3) Einkommensrisiken: Mit den neuen Formen flexibler Arbeitszeit und der Drohmacht von Unternehmen, Standorte zu schließen, sind Einkommensrisiken verbunden. Arbeit nach Bedarf kann zu Einkommensschwankungen führen, Arbeit nach Zielvereinbarungen hat häufig nicht entgoltene Überstunden zur Folge (Lehndorff 2007). Die zahlreichen Betriebsvereinbarungen zur Standortsicherung und zur Sicherung der Beschäftigung werden immer wieder mit Einkommensverzichten der Beschäftigten bezahlt (Streeck/Rehder 2003).

(4) Beschäftigungsrisiken: Die interne Flexibilisierung schützt schließlich nicht vor Beschäftigungsrisiken. Selbst florierende Unternehmen setzen die Ankündigung von Stellenabbau ein, um den Aktienkurs in die Höhe zu treiben. Eine Belastung eigener Art stellen die häufigen (und für die Betroffenen oft nicht nachzuvollziehenden) Umstrukturierungen der Unternehmen dar. Die Ausgliederung von Geschäftsbereichen, der Verkauf von Unternehmenssparten, wenn nicht gar des gesamten Unternehmens, haben in aller Regel zur Folge, dass Arbeitsplätze zur Disposition gestellt werden (Flecker 2007, S. 8; Schumann in diesem Heft).

Die Verbindung zwischen Verunsicherung und Ungerechtigkeitsempfinden hat hier eine starke Quelle: in der Erfahrung vieler Angehöriger der lohn- und gehaltsabhängigen Mitte, dass sie in ihrer Erwerbsarbeit zwar immer stärker individuell dafür verantwortlich gemacht werden, ihre Leistung zu erhöhen und ihren Arbeitsplatz zu er- 
halten - selbst unter materiellen Verzichten und zeitlichen Opfern; dass ihnen aber gleichwohl die Gewissheit vorenthalten wird, mit Anerkennung, Aufstieg und beruflicher Sicherheit rechnen zu können.

\subsection{EROSION VON STATUS- SICHERHEIT DURCH UMBAU DES SOZIALSTAATS}

Der Wandel der Arbeits- und Beschäftigungsverhältnisse wird seit den 1980er Jahren von weitreichenden Veränderungen im Kernbereich des Sozialstaats, dem Arbeitsund Sozialleistungsrecht, teils vorangetrieben, teils flankiert. Blanke und Bleses sprechen vom „Abschied von der Vorstellung einer - im Vergleich zu anderen Gruppen besonderen Schutzbedürftigkeit des Arbeitnehmers" (Blanke/Bleses 2007, S. 366). Sie zeichnen nach, wie aus dem Arbeitsrecht schrittweise der Schutz des erreichten Erwerbsstatus verschwindet und im Sozialleistungsrecht der Bezug zum Einkommen aus Erwerbstätigkeit eingeschränkt wird. Der Wechsel von der Arbeitslosenhilfe zum Arbeitslosengeld II auf Sozialhilfeniveau ist ein gutes Beispiel für eine solche Einschränkung. Rechtliche Absicherungen der Erwerbsposition und der an sie geknüpften Ansprüche werden zurückgenommen, an Stelle einer Status- und Lebensstandardsicherung wird nach und nach eine statusunabhängige Grundsicherung auf bescheidenerem Niveau aufgebaut. Für die Lohnabhängigen hat dies eine doppelte Verunsicherung zur Folge: Verunsicherung hinsichtlich der beruflichen Zukunft und hinsichtlich der materiellen Folgen von Krankheit, Arbeitslosigkeit und Alter.

Somit findet eine Verschiebung in der Strukturierung von sozialer Ungleichheit statt: von der sozialstaatlichen Absicherung unterschiedlicher Positionen des Erwerbsstatus und der mit ihnen verknüpften Lebensniveaus über die einzelnen Lebensphasen hinweg hin zu einer Ungleichheit, die unvermittelter auf Marktchancen und -risiken, somit Marktmacht beruht, abgefedert durch sozialstaatliche Grundsicherungen. Die Mehrheit der Bevölkerung und mit ihr die lohn- und gehaltsabhängige Mitte reagiert auf die zunehmende Entsicherung der Lebensperspektiven mit Un- gerechtigkeitsempfindungen. Darin artikuliert sich, wie Neckel schreibt, ,auch die Enttäuschung eines sozialmoralischen Bewusstseins, das Gelegenheit hatte, in den langen Jahrzehnten des rheinischen Kapitalismus starke Inklusionserwartungen auszubilden, die nun ganz offensichtlich hinfällig geworden sind“ (Neckel 2008, S. 39f.). Bis heute richten sich diese Erwartungen, wie regelmäßige Umfragen zeigen, bei der großen Bevölkerungsmehrheit nach wie vor an den Sozialstaat.

\section{Perspektiven für eine Politik der Reduzierung von sozialer Ungleichheit und Unsicherheit}

In der Fortdauer der Inklusionserwartungen liegt eine Chance, die „Mitte“ für eine Politik der Inklusion auf neuer Grundlage zu gewinnen. In der Enttäuschung der Inklusionserwartungen liegt aber auch die Gefahr von Entsolidarisierungen. Hält die gegenwärtige Tendenz der Verschiebung hin zur Ungleichheit durch Marktmacht an, dann droht Entsolidarisierung vor allem von zwei Seiten: zum einen von Seiten derer, die sich stark genug fühlen, auf diese Karte zu setzen; zum anderen von denjenigen, die sich von sozialem Abstieg bedroht sehen. Die einen dürften sich dann mehr denn je für Kürzungen der öffentlichen Ausgaben, Reduzierung von Steuern und Abgaben und die weitere Privatisierung sozialer Dienstleistungen einsetzen (zu den Gefahren einer solchen Politik vgl. Bofinger in diesem Heft). Die anderen hingegen dürften sich in einer Konkurrenz um knappe Ressourcen mit all denen positionieren, die bereits abgestiegen sind oder als ,Fremde" Ansprüche erheben. Gerade wegen der aufgezeigten Zwiespältigkeiten in der sozialstrukturellen Mitte ist die Entwicklung noch offen (Kadritzke 2006).

Die Gefahr der Entsolidarisierung wird allerdings durch die derzeit vorherrschende Politikrichtung verstärkt. Eine Politik, die immer wieder Eigenverantwortung propagiert und damit nichts anderes als die Aufforderung meint, Marktrisiken zu überneh- men und sich am Markt zu versichern, fordert die Bessergestellten geradezu heraus, sich mental und schließlich auch politisch von den solidarischen Sicherungssystemen zu verabschieden - zumal dann wenn deren Leistungen zurückgehen.

Ein Blick über die Grenzen zeigt, dass Länder mit umverteilenden Sozialstaaten, die soziale Ungleichheiten reduzieren, eher in der Lage sind, Flexibilität und Sicherheit miteinander zu verbinden, als Länder mit ausgeprägteren sozialen Ungleichheiten (Auer 2007; Schmid in diesem Heft). Eine Politik in diese Richtung würde in Deutschland einen drastischen Kurswechsel bedeuten. Sie würde erfordern, die sozialen Sicherungssysteme auf eine neue, erweiterte finanzielle Grundlage zu stellen und dabei gezielt die Schieflage der Einkommensund Vermögensverteilung zu korrigieren. Sie käme auch nicht umhin, endlich die durch das Bildungssystem reproduzierten sozialen Ungleichheiten anzugehen (vgl. hierzu auch Allmendinger/Helbig in diesem Heft) und elementare soziale Bürgerrechte (Gesundheitsversorgung, Alterssicherung) unabhängig von den Wechselfällen der Erwerbsbiografien zu stärken.

Ließe sich für eine Politik der Reduzierung von sozialer Ungleichheit und Unsicherheit die Mehrheit der lohn- und gehaltsabhängigen Mitte gewinnen? Das lässt sich allein schon deshalb nicht beantworten, weil die Auseinandersetzung um eine solche Politik noch gar nicht ernsthaft geführt worden ist. Sie hätte neben dem bereits genannten Argument, dass es ohne sozial-materielle Sicherheiten kein selbstbestimmtes Handeln geben kann, in jedem Fall ein weiteres auf ihrer Seite: die Lebensqualität. Länder mit ausgleichenden und Lebensperspektiven gewährleistenden sozialen Sicherungssystemen weisen eine höhere Lebenszufriedenheit auf (Böhnke/ Kohler 2007). Egalitärere Gesellschaften, deren Ungleichheiten auch noch als gerecht betrachtet werden, können bessere Gesundheitswerte und tragfähigere soziale Beziehungen verbuchen als weniger egalitäre (Wilkinson 2004). Aus epidemiologischer Sicht hat dies einen entscheidenden Grund: Sie erzeugen weniger soziale Ängste. 
Alda, H. (2005): Beschäftigungsverhältnisse, in: SOFI/IAB/ISF/INIFES (Hrsg.): Berichterstattung zur sozioökonomischen Entwicklung in Deutschland. Arbeit und Lebensweisen. Erster Bericht, Wiesbaden, S. 245-269

Andreß, H.-J./Kronauer, M. (2006): Arm - Reich, in: Lessenich, S./Nullmeier, F. (Hrsg.): Deutschland - eine gespaltene Gesellschaft, Frankfurt am Main, New York, S. 28-52

Auer, F. (2007): Security in labour markets: combining flexibility with security for decent work. ILO Economic and Labour Market Papers 12 Genf

Bigalke, S. (2007): Deutsche glauben nicht mehr an Chancengleichheit, in: Spiegel online, 13. September

Blanke, T./Bleses, P. (2007): Flexicurity im Arbeits- und Sozialleistungsrecht, in: Kronauer, M./Linne, G. (Hrsg.): Flexicurity. Die Suche nach Sicherheit in der Flexibilität, Berlin, S. 365-384

Böhnke, P. (2006): Am Rande der Gesellschaft. Risiken sozialer Ausgrenzung, Opladen

Böhnke, P./Kohler, U. (2007): Determinanten des Glücks: Lebenszufriedenheit in Europa, in: WSI-Mitteilungen 7, S. 373-379

Bourdieu, P./Boltanski, L./de Saint Martin, M. (1981): Kapital und Bildungskapital. Reproduktionsstrategien im sozialen Wandel, in: Bourdieu, P./Boltanski, L./de Saint Martin, M./Maldidier, P.: Über die Reproduktion sozialer Macht, Frankfurt am Main, S. 23-87

Castel, R. (2000): Die Metamorphosen der sozialen Frage, Konstanz Ehrenberg, A. (2003): Das erschöpfte Selbst, Frankfurt a. M., New York Flecker, J. (2007): Interne Flexibilisierung - von der Humanisierungsvermutung zum Risikobefund, in: Kronauer, M./Linne, G. (Hrsg.): Flexicurity. Die Suche nach Sicherheit in der Flexibilität, Berlin, S. 73-93

Friedrich-Ebert-Stiftung (2006): Gesellschaft im Reformprozess. Die Friedrich-Ebert-Stiftung untersucht Reformbereitschaft der Deutschen. FES Gesellschaft im Reformprozess komplett.pdf

Geiger, T. (1932/1967): Die soziale Schichtung des deutschen Volkes. Soziographischer Versuch auf statistischer Grundlage, Stuttgart Grabke, M./Frick, J. R. (2008): Schrumpfende Mittelschicht - Anzeichen einer dauerhaften Polarisierung der verfügbaren Einkommen? Wochenbericht 10 des DIW Berlin, Berlin

Groß, M. (2007): Soziale Sicherheit und Gerechtigkeit, in: Berliner Journal für Soziologie, Band 17, Teil 2, S. 151-166

Kadritzke, U. (2004): White-Collar Blues. Über Angestellte im modernen und im neuesten Kapitalismus, in: Artus, I./Trinczek, R. (Hrsg.): Über Arbeit, Interessen und andere Dinge. Phänomene, Strukturen und Akteure im modernen Kapitalismus, München, Mering, S. 101-121
Kadritzke, U. (2006): Kein Platz mehr im letzten Flugzeug. Die Mittelklassen in der Zone der Verwundbarkeit, in: Le Monde diplomatique, Dezember, S. 12-13

Kaufmann, F. X. (2003): Sicherheit: Das Leitbild beherrschbarer Komplexität, in: Lessenich, S. (Hrsg.): Wohlfahrtsstaatliche Grundbegriffe. Historische und aktuelle Diskurse, Frankfurt am Main, New York, S. 73-104

Kazim, H. (2007): Jeder Dritte findet seine Arbeit schrecklich, in: Spiegel online, 11. September

Keller, B./Seifert, H. (2007): Atypische Beschäftigungsformen und Flexicurity, in: Kronauer, M./Linne, G. (Hrsg.): Flexicurity. Die Suche nach Sicherheit in der Flexibilität, Berlin, S. 127-147

Klammer, U. (2007): Flexicurity aus der Perspektive des Lebensverlaufs, in: Kronauer, M./Linne, G. (Hrsg.): Flexicurity. Die Suche nach Sicherheit in der Flexibilität, Berlin, S. 249-273

Kronauer, M. (2007): Neue soziale Ungleichheiten und Ungerechtigkeitserfahrungen: Herausforderungen für eine Politik des Sozialen, in: WSIMitteilungen 7, S. 365-372

Lehndorff, S. (2007): Arbeitszeitreduzierung unter Druck, in: Kronauer, M./Linne, G. (Hrsg.): Flexicurity. Die Suche nach Sicherheit in der Flexibilität, Berlin, S. 205-220

Neckel, S. (2008): Die gefühlte Unterschicht. Vom Wandel der sozialen Selbsteinschätzung, in: Lindner, R./Musner, L. (Hrsg.): Unterschicht. Kulturwissenschaftliche Erkundungen der "Armen“ in Geschichte und Gegenwart, Freiburg i. Br., Berlin, Wien, S. 19-40

Sen, A. (2002): Ökonomie für den Menschen. Wege zu Gerechtigkeit und Solidarität in der Marktwirtschaft, München

Spiegel online (2007): Deutsche vermissen soziale Gerechtigkeit. 10. Dezember

Statistisches Bundesamt (2006): Datenreport 2006. Zahlen und Fakten über die Bundesrepublik Deutschland, Bonn.

Streeck, W./Rehder, B. (2003): Der Flächentarifvertrag: Krise, Stabilität und Wandel, in: Industrielle Beziehungen 3, S. 341-362

Vogel, B. (2006): Sicher - Prekär, in: Lessenich, S./Nullmeier, F. (Hrsg.): Deutschland - eine gespaltene Gesellschaft, Frankfurt am Main, New York, S. 73-91

Wilkinson, R. G. (2004): Gesundheit, Hierarchie und soziale Angst, in: Zilian, G. (Hrsg.): Insider und Outsider, München, Mering, S. 119-143 Wright, E. O. (1997): Class counts. Comparative studies in class analysis, Cambridge. 